\title{
Augmentation of the early component of the human blink reflex with closely spaced stimulus pairs
}

\author{
GEOFF HAMMOND and YVONNE PLANT \\ University of Western Australia, Nedlands, Western Australia, Australia
}

\begin{abstract}
The effect of closely spaced percutaneous stimuli (S1 and S2) on the early (R1) component of the human blink reflex was studied in three experiments. In all experiments, successive R1 components (called $\mathrm{R}_{1}$ and $\mathrm{R} 1_{2}$ here) were frequently elicited by $\mathrm{S} 1-\mathrm{S} 2$ pairs with stimulus onset asynchronies (SOAs) as brief as $4 \mathrm{msec}$. The successive R1s appeared to be under the separate control of S1 and S2, respectively. Experiment 1 (in which S1 was set at each subject's threshold for R1 elicitation and S2 was set at $80 \%$ of that threshold) and Experiment 2 (in which S1 was set at $80 \%$ of each subject's threshold for R1 elicitation and S2 was set at the threshold level) showed that $\mathrm{S} 2$ had no effect on either the probability or the amplitude of $\mathrm{R} 1_{1}$ but that $\mathrm{S} 1$ increased both the probability and the amplitude of $R 1_{2}$ above control levels established by presentation of $\mathrm{S} 2$ alone. Augmentation of $\mathrm{R}_{2}$ was greatest at the shortest $\mathrm{SOA}$ tested (4 msec) and decayed to zero at an SOA of about 11-12 msec. Paired stimuli produced no systematic effect on the latency of either $R 1_{1}$ or $R 1_{2}$. Experiment 3 showed that the probability and amplitude of $\mathrm{R} 1_{1}$ increased with $\mathrm{S} 1$ intensity but were unaffected by $\mathrm{S} 2$ intensity over the range tested. The probability and amplitude of $\mathrm{R} 1_{2}$ increased with $\mathrm{S} 2$ intensity and were increased by the presence of S1; the augmentation produced by S1 was greater at the 4- than at the 8-msec SOA, and increased with S2 intensity. There were no systematic effects on the latency of either R1. Reflex augmentation in these experiments is not consistent with the model of temporal integration of stimulus energy. Instead, a percutaneous stimulus appears to evoke a transient excitation of the R1 pathway; a second percutaneous stimulus presented before the excitation decays is more likely to elicit an R1 than otherwise, and the $\mathrm{R} 1$ elicited will on the average be larger.
\end{abstract}

The amplitude and latency of the human blink reflex are not fixed by the parameters of the blink-eliciting stimulus, but are sensitive to environmental variables such as stimuli that precede or follow the blink-eliciting stimulus (Plant \& Hammond, 1989; Sanes \& Ison, 1979) and subjective variables such as attentiveness (Hackley \& Graham, 1984), anticipation of the reflex-eliciting stimulus (Ison, Sanes, Foss, \& Pinckney, 1990), and emotional state (Vrana, Spence, \& Lang, 1988). The sensitivity of the human blink reflex to such variables, together with its ease of elicitation and measurement, makes it a useful model system. The compensatory changes in the blink reflex in different adapting conditions noted by Evinger and Manning (1988) led them to propose it as a model system for motor learning. In particular, the cutaneous blink reflex (usually elicited by electrical stimulation of the supraorbital nerve or a mechanical tap to the forehead) has two successive electromyographic components, a brief short-latency component mediated by a probable disynaptic pathway (R1), and a more prolonged later

This research was supported by a grant from the Australian Research Council to G.H. Send correspondence to G. Hammond, Department of Psychology, University of Western Australia, Nedlands, WA 6009, Australia (e-mail: jeff@psy.uwa.edu.au) component mediated by a polysynaptic pathway (R2). This feature of the cutaneous blink reflex allows separate examination of the effects of environmental and subjective variables on pathways of different complexity, extending its value as a model system. In the present experiments, we observed the effects of closely spaced paired stimuli on the amplitude and latency of R1 to determine how stimulus energy is integrated in the simple pathway that mediates this component.

The amplitude of $R 1$ is greater with paired than with single percutaneous stimuli (Hammond \& Dennon, 1991). Hammond and Dennon (1991) interpreted the augmentation of R1 seen in these conditions as an instance of temporal integration of stimulus energy, with the second stimulus adding to the size of the response elicited by the first. Augmentation increased with the intensity of the second stimulus and was present when the second stimulus itself was below threshold for reliable elicitation of R1. Substantial augmentation was seen at all stimulus onset asynchronies (SOAs) tested $(1,2,4$, and $8 \mathrm{msec}$ ), with only slight decay over the three longest SOAs. Hammond and Dennon also noted an apparent latency shift of $\mathrm{R} 1$ : on about one third of the paired-stimulus presentations with an SOA of 4 or $8 \mathrm{msec}$, the latency of R1 (which was scored by visual identification of the component on a storage oscilloscope) was delayed by an amount equal to the 
SOA on that trial. These "delayed" responses were augmented as much as the normal-latency responses.

The aims of the first experiment were to determine more fully the time course of R1 augmentation found with percutaneous stimulus pairs and to examine the form and latency of the response in more detail. Paired percutaneous stimuli with the first stimulus at threshold for reliable R1 elicitation and the second stimulus below this intensity were used.

\section{EXPERIMENT 1}

\section{Method}

Subjects. Eight volunteers ( 4 males and 4 females) were subjects. Their ages ranged from 18 to 46 with a median of 26 years. The subjects had no history of neurological disorders.

Apparatus and Procedure. Miniature stainless steel stimulating electrodes were attached to the skin over the left supraorbital nerve. One electrode was placed immediately above the eyebrow in line with the supraorbital notch, with the other about $1 \mathrm{~cm}$ superior. EMG activity from the ipsilateral orbicularis oculi muscle was recorded with $1-\mathrm{cm}$-diameter gold-plated electrodes. One recording electrode was placed at the lateral canthus with the other below the lower lid margin in line with the pupil with forward gaze. A ground electrode was placed on the ispilateral mastoid process. The impedance between the recording electrodes ranged from 5 to $10 \mathrm{k} \Omega$ for all subjects. EMG was amplified with a Grass 7P511 amplifier with half-amplitude high-pass and low-pass frequencies of $3 \mathrm{~Hz}$ and $3 \mathrm{kHz}$, respectively.

The subjects were seated in a comfortable reclining chair alone in a sound-attenuated room and were asked to relax and close their eyes, but not fall asleep. Thresholds for the blink elicitation were then determined. The eliciting stimuli were $0.2-\mathrm{msec}$ biphasic square-wave shocks produced by a Grass SD9 stimulator and routed through a Grass constant-current unit. Single stimuli were applied to the supraorbital nerve at intertrial intervals of between about 10 and $20 \mathrm{sec}$, beginning at $05 \mathrm{~mA}$ and increasing in steps of about $0.5 \mathrm{~mA}$ until the second component (R2) of the blink reflex was elicited. The intensity was then increased in steps of about $0.2 \mathrm{~mA}$ until a reliable first component (R1) was elicited on three successive stimulus presentations. Reliable R1 elicitation threshold intensities for the 8 subjects ranged from 3.6 to $12.5 \mathrm{~mA}$, with a mean of $7.6 \mathrm{~mA}$.

Once thresholds were determined, the subjects were told that the stimuli would be presented at intervals of between 10 and $30 \mathrm{sec}$ and that they should restrict any movements to the few seconds immediately after a stimulus presentation. Pairs of reflex-eliciting stimuli (denoted S1 and S2, respectively) were presented at SOAs of $4,8,9,10,11,12,13$, and $14 \mathrm{msec}$. The intensity of S1 was set at the subject's R1 elicitation threshold, and the intensity of S2 was set proportionately at 0.8 of that threshold. Trials of single percutaneous stimuli at both intensities (S1-alone and S2-alone trials) were given to establish control reflex values. Nine of the different stimulus conditions (eight $\mathrm{S} 1-\mathrm{S} 2$ stimulus pairs and the S1-alone presentations) were delivered randomly in each of eight trial blocks for a total of 72 trials; eight S2-alone presentations were given after the other trials were completed. The intertrial interval varied from 10 to $32 \mathrm{sec}$, with a mean of $18 \mathrm{sec}$.

Onset latency and peak-to-peak amplitude of R1 were scored directly from the amplified tracings displayed on a digital storage oscilloscope. The sweep speed on the oscilloscope allowed latency to be scored to the nearest millisecond. Reflex amplitudes were later converted to signal amplitude in millivolts. Trials contaminated with EMG activity in the $10 \mathrm{msec}$ before onset of the first stimulus were discarded, leading to the loss of $2.3 \%$ of all trials.
Because scoreable responses were not always elicited, separate measures of response probability and response amplitude are reported. Response probability was estimated from the frequency of scoreable responses, and response amplitude was scored as the mean amplitude of the scoreable responses; trials on which a response was not detected were not included in the calculation of the mean amplitude (i.e., zero values did not enter into the calculation of the means). The results are reported as mean response probabilities, amplitudes, and latencies with associated standard errors. The influence of the independent variables are estimated with $\omega^{2}$, the proportion of variance accounted for by the variable in question.

\section{Results and Discussion}

Figure 1 shows sample EMG traces obtained with single-stimulus presentation and paired-stimulus presentation at each SOA tested. With paired stimuli, a frequent differentiation of the early response into two successive

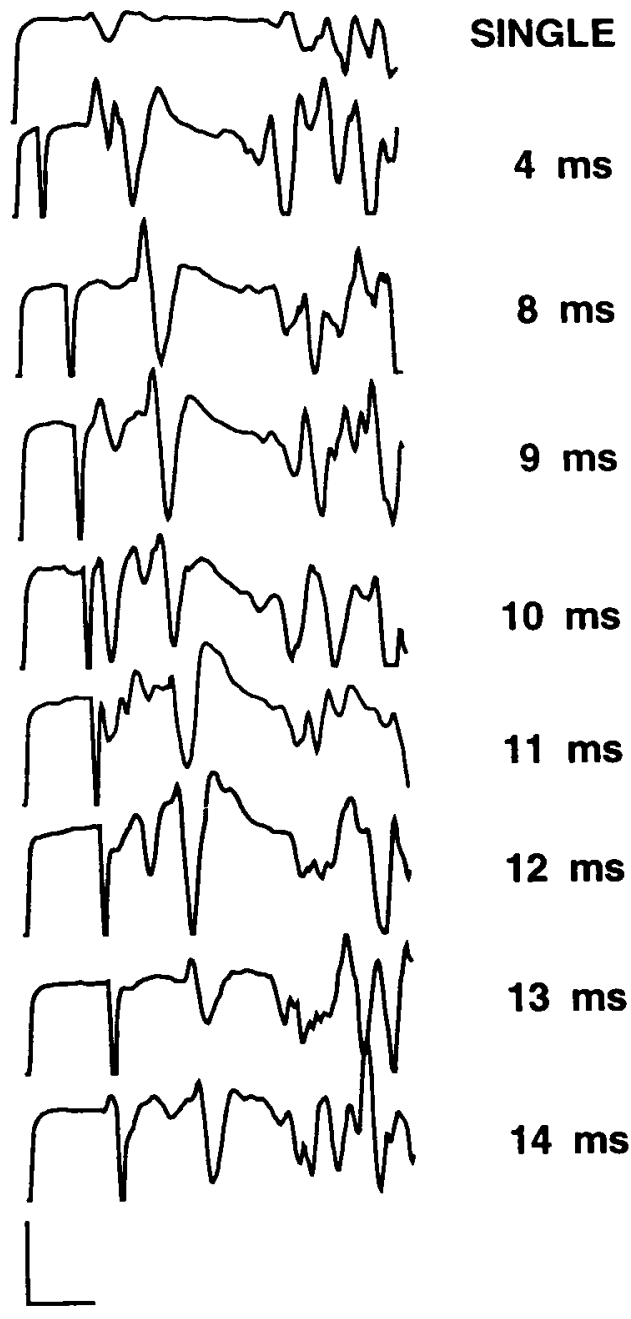

Figure 1. EMG tracings from a single-stimulus presentation and from paired-stimulus presentations at each stimulus onset asynchrony tested in Experiment 1. Negative-going stimulus artifact is evident on the left of each tracing. The start of the $\mathbf{R 2}$ component is present on the right of each tracing. The vertical and horizontal calibration bars show $0.5 \mathrm{mV}$ and $10 \mathrm{msec}$, respectively. 
$\mathrm{R} 1$ components (called here $\mathrm{R} \mathrm{l}_{1}$ and $\mathrm{R} \mathrm{l}_{2}$ ) was observed. Figure 2 shows EMG traces from two different trials with a 10 -msec SOA to illustrate a case in which only $R l_{2}$ was present (upper trace) and a case in which both $R 1_{1}$ and $R 1_{2}$ were present (lower trace). Successive $R 1_{1}$ and $R 1_{2}$ responses were seen on $50.0 \%$ of the scoreable pairedstimulus trials on which $R 1$ activity was detectable. In all cases in which two R1s appeared, they were separated by an interval equal to the SOA of the paired stimuli, implying that $R 1_{1}$ was elicited by the first stimulus of the pair and that $R 1_{2}$ was elicited by the second stimulus of the pair. Assuming this, the appropriate control values for probability, amplitude, and latency for $R 1_{1}$ are those established by presentation of the first stimulus alone, and for $R 1_{2}$, those established by presentation of the second stimulus alone.

Figure 3 shows mean response probability for the R 1 recorded with the single-stimulus presentations and for $R 1_{1}$ and $R 1_{2}$ recorded with $S 1-S 2$ pairs at each SOA. An R1 was not always elicited on S1-alone trials and was sometimes elicited on S2-alone trials; the probabilities of $\mathrm{R} 1$ occurrence were $0.89(S E M=.04)$ and $0.27(S E M=$ .09 ) for S1-alone and S2-alone presentations, respectively. Two successive RIs were not always present with paired stimulus presentations. The probability of occurrence of an $R 1_{1}$ with paired stimuli was close to the $S 1$-alone probability except at the 11-, 12-, and 13-msec SOAs, where it was lower. The probability of occurrence of an $\mathrm{Rl}_{2}$ was greater than S2-alone probabilities at short SOAs and declined systematically with increasing SOA to the singlestimulus levels at the 12-msec SOA. SOA was an influen-

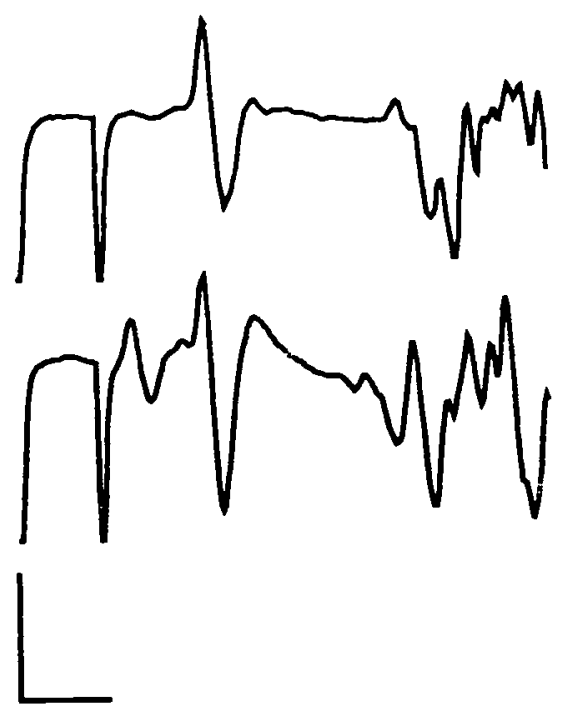

Figure 2. EMG tracings from paired-stimulus presentations at the 10-msec stimulus onset asynchrony. The top trace shows an $\mathbf{R 1}_{2}$ with no preceding $R 1_{1}$, and the bottom trace shows both the $R 1_{1}$ and the $R_{2}$ responses. Negative-going stimulus artifact is evident on the left of each tracing. The start of the $R 2$ component is present on the right of each tracing. The vertical and horizontal calibration bars show $0.5 \mathrm{mV}$ and $10 \mathrm{msec}$, respectively.

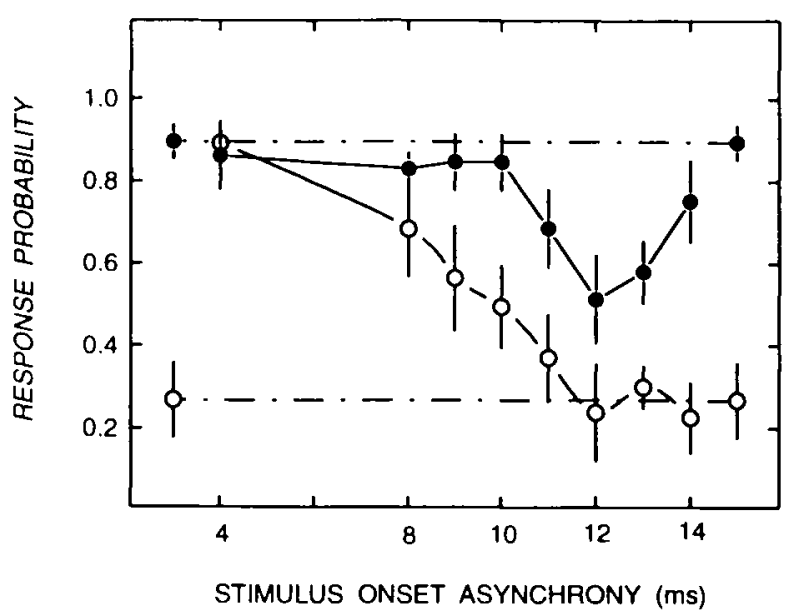

Figure 3. Mean response probability of $R 1_{1}$ (filled symbols) and $\mathbf{R 1}_{2}$ (open symbols) as a function of the stimulus onset asynchrony of paired stimuli in Experiment 1. The mean response probabilities for the S1-alone and S2-alone presentations are shown by the broken lines connecting the closed and open symbols, respectively. Error bars show \pm 1 SEM.

tial determinant of probability for both successive responses (estimated $\omega^{2}=.30$ and .60 for $R 1_{1}$ and $R l_{2}$, respectively) ${ }^{1}$

The mean peak-to-peak amplitudes of the singlestimulus R1s and of each R1 with paired stimuli at the different SOAs are shown in Figure 4. The effect of the different intensities of $\mathrm{S} 1$ and $S 2$ is evident in the mean response amplitudes with single-stimulus presentations. With paired stimuli, the mean amplitude of $R 1_{1}$ showed little variation with SOA and was close to the mean S1alone response amplitude at all SOAs (estimated $\omega^{2}$ for SOA $=.00$ ). The amplitude of $R 1_{2}$ was in contrast a decreasing function of SOA, reaching a stable asymptote at an SOA of about $11 \mathrm{msec}$ (estimated $\omega^{2}$ for SOA = .63 ). $R 1_{2}$ was augmented relative to $S 2$-alone amplitude at brief SOAs; the mean amplitudes were 6.7 and 3.5 times the S2-alone level at the 4- and 8-msec SOAs, respectively.

The latency of $R 1_{1}$ was recorded from the onset of the first stimulus, and the latency of $R 1_{2}$ was recorded from the onset of the second stimulus. R1 latency for S1-alone presentation was $11.0 \mathrm{msec}(S E M=0.21)$ and for S2alone presentation was $12.0(S E M=0.50)$. There was no effect of SOA on the latency of either $R 1_{1}$ or $R 1_{2}$ with paired stimuli. The mean latency of $R 1_{1}$ with paired stimuli was $11.3 \mathrm{msec}(S E M=0.11)$ and the mean latency of $R 1_{2}$ with paired stimuli was $11.7 \mathrm{msec}(S E M=0.17)$. The similarity of the latencies of the two successive R1 components to those of the responses elicited by singlestimulus presentations support the view that they were controlled by the first and second stimuli, respectively.

Neither the probability nor the amplitude of $R 1_{1}$ was affected by the presence of the second stimulus at any of the SOAs tested. In contrast, both the probability and the 


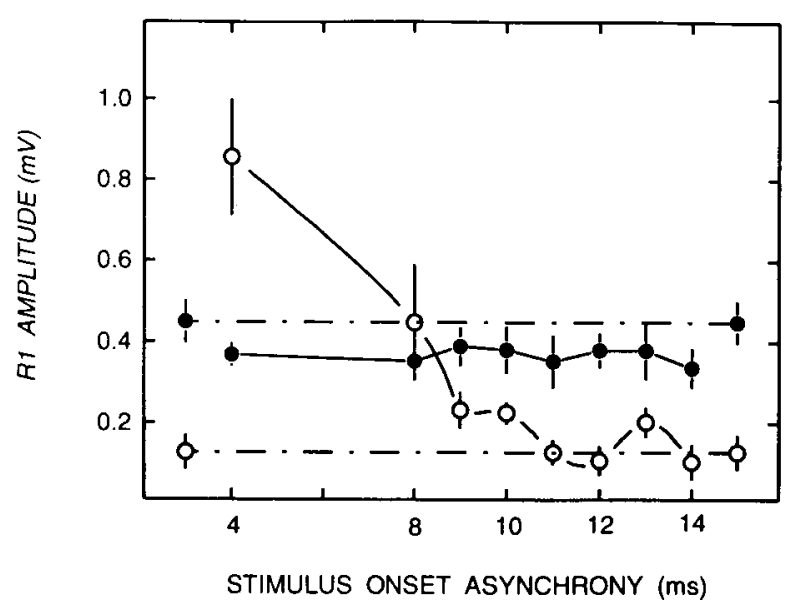

Figure 4. Mean response amplitude (in millivolts) of $\mathrm{R1}_{1}$ (filled symbols) and $\mathrm{RI}_{2}$ (open symbols) as a function of the stimulus onset asynchrony of paired stimuli in Experiment 1. The mean response amplitudes for the S1-alone and S2-alone presentations are shown by the broken lines connecting the closed and open symbols, respectively. Error bars show \pm 1 SEM.

amplitude of $R 1_{2}$ were affected systematically by the presence of the first stimulus; $R 1_{2}$ was both more probable and larger at brief SOAs, and this augmentation declined systematically with SOA, reaching zero at an SOA of about $11 \mathrm{msec}$. The similarity of the amplitude and latency of $R 1_{1}$ with paired stimuli to the $R 1$ elicited by $S 1$ alone and the decline of the probability and amplitude of $R 1_{2}$ at the long SOAs to the corresponding values for the R1 elicited by $S 2$ alone (see Figures 3 and 4 ) support the idea that $R 1_{1}$ and $R l_{2}$ are under the independent control of the two successive stimuli of a pair.

These results confirm in general Hammond and Dennon's (1991) report that $R 1$ amplitude is augmented with paired percutaneous stimuli presented with brief SOAs. Augmentation of R1 in these stimulus conditions indicates that the R1 pathway is subject to an elementary form of reflex modification. These results also clarify the nature of $\mathrm{R} 1$ augmentation and the conditions under which it occurs. First, what appeared to Hammond and Dennon as a latency shift of a single R1 with S1-S2 stimulus pairs separated by 4 and $8 \mathrm{msec}$ is instead two separate responses, with the second response larger than the first. Because Hammond and Dennon also scored the latency of the slower R2 (which had a mean latency of about $35 \mathrm{msec}$ ) from the oscilloscope trace of the EMG, a relatively slow sweep speed was necessary. This slow sweep speed did not allow resolution of the two nearly contiguous R1 responses. Second, temporal integration of stimulus energy (which has been the usual account of reflex augmentation with paired stimuli at brief SOAs) is not an adequate model of the stimulus interactions shown in $\mathrm{R} 1$ amplitude. This model implies integration of the energy of the second stimulus with that of the first, reflexeliciting, stimulus, thereby augmenting the response elicited by that stimulus. The amount of temporal integration is a decreasing function of the delay of the second stimulus, reaching zero after some interval that defines the integration window. Given the assumption that two R1 responses are elicited successively by the successive stimuli, the present results show that the second stimulus does not affect the size of the response elicited by the first; as noted above, the suppression of the probability of $R 1_{1}$ at some SOAs was probably a measurement artifact. By the usual definition, temporal integration does not occur. Instead, the mechanism of R1 augmentation is found in the effect of the first stimulus on the response elicited by the second stimulus. An R1 initiated by an adequate stimulus is unaffected by a second percutaneous stimulus. Instead, the presence of the first stimulus increases the excitability of the R1 pathway so that a second (subthreshold) stimulus elicits an R1 more frequently than it otherwise would and the amplitude of the R1 elicited by the second stimulus is larger than it otherwise would be.

\section{EXPERIMENT 2}

Augmentation of the probability and amplitude of $\mathrm{R} 1_{2}$ in Experiment $\mathbf{l}$ decayed to an asymptote of the S2-alone level at an SOA of about 11-12 msec. This time course, however, may have been determined not by the SOA, but by the interval between the successive responses, which was necessarily confounded with SOA. The $R 1_{2}$ may have been augmented by processes associated with emission of $\mathrm{R} 1_{1}$ rather than the presentation of S1. In Experiment 2, we therefore examined whether augmentation of $R 1_{2}$ with paired stimuli was present when the first stimulus did not always elicit a detectable $\mathrm{R} 1$. The procedure was the same as for Experiment 1, except that the intensity of the first stimulus in the pair (S1) was below the intensity threshold for reliable elicitation of R1, and the second stimulus (S2) was at the elicitation threshold for R1.

\section{Method}

Subjects. Six volunteers were selected from the same population, with the same criteria, as in Experiment 1. There were 3 males and 3 females, from 19 to 46 years old, with a median age of 20 years. Three of the subjects had served in Experiment 1 .

Apparatus and Procedure. The procedures were similar to those in Experiment 1. The impedance between the recording electrodes ranged from 5 to $12 \mathrm{k} \Omega$ for all subjects. Thresholds for reliable elicitation of $\mathbf{R} 1$ were determined in the same way as in Experiment 1. These threshold intensities ranged from 4.7 to $11.8 \mathrm{~mA}$, with a mean of $7.9 \mathrm{~mA}$.

Pairs of reflex-eliciting stimuli were presented at SOAs of 4,8 , $9,10,11,12,13$, and 14 msec. The intensity of the first stimulus was set at 0.8 of the subject's reliable elicitation threshold level, and the intensity of the second stimulus, at the elicitation threshold. Single percutaneous stimuli at both intensities were given to establish control reflex values. The presentation sequence of each stimulus pair and the single stimuli was random within each of eight blocks. A total of 80 trials were given. The intertrial interval varied from 10 to $32 \mathrm{sec}$, with a mean of $17 \mathrm{sec}$.

Trials contaminated with EMG activity in the $10 \mathrm{msec}$ before $\mathrm{S} 1$ onset were rejected; $2.1 \%$ of all trials were discarded. 


\section{Results and Discussion}

Two successive R1 components were seen on $57.0 \%$ of the paired-stimulus trials on which at least one $R I$ was detected. The probabilities of occurrence of $R 1_{1}$ and $R 1_{2}$ with paired stimuli at each SOA and with the singlestimulus presentations are shown in Figure 5. The probability of $R 1_{1}$ did not vary systematically with the SOA of the paired stimuli, and was close to the S1-alone probability at all SOAs. Similar results were observed for $R \mathbf{l}_{2}$, although the probability was increased slightly above the S2-alone level at the 4- and 8-msec SOAs. The high probability of $\mathrm{R} 1$ occurrence with $\mathrm{S} 2$ alone $(.84 ; S E M=.07)$ left little room for the probability of elicitation of this component to increase.

The mean peak-to-peak amplitudes for each R1 with paired stimuli at each SOA and for the single-stimulus presentations are shown in Figure 6. As in Experiment 1, the size of $R 1_{1}$ did not vary systematically with SOA and was close to the mean amplitude obtained with $\mathrm{S} 1$-alone presentations. In contrast, the mean amplitude of $R 1_{2}$ was largest with the shortest SOA and declined systematically with increasing SOA toward the mean amplitude obtained with S2-alone presentation.

The mean latency for both S1-alone and S2-alone presentations was $11.8 \mathrm{msec}(S E M=0.53$ and 0.33 , respectively). The mean latency of both $R 1_{1}$ and $R 1_{2}$ with paired stimuli was $11.8 \mathrm{msec}(S E M=0.17$ and 0.15 , respectively).

These results confirm those found in the first experiment: first, two successive Rl components were often elicited with paired stimulus presentations at brief SOAs; second, the presence of a second stimulus did not affect the probability or amplitude of $R 1_{1}$; third, the presence of the first stimulus increased the amplitude of $\mathrm{R}_{2}$; and fourth, augmentation of the amplitude of $R 1_{2}$ by the lead-

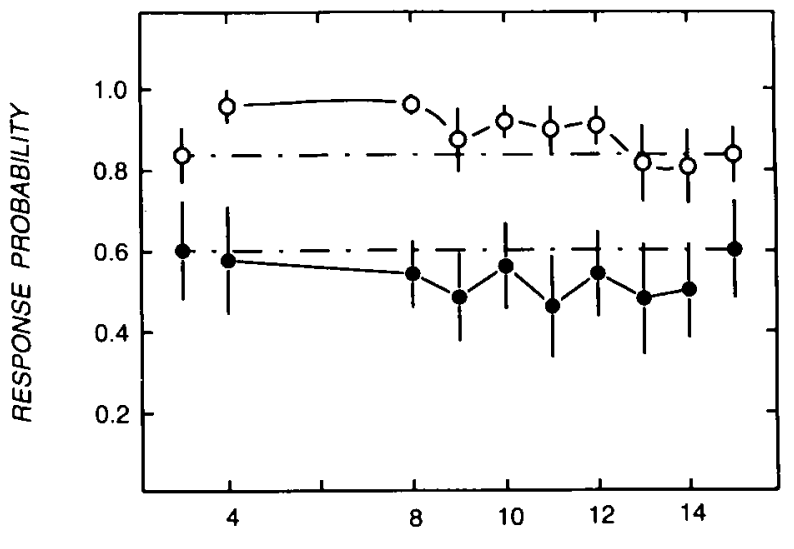

STIMULUS ONSET ASYNCHRONY (ms)

Figure 5. Mean response probability of $\mathrm{R1}_{1}$ (filled symbols) and $\mathrm{Rl}_{2}$ (open symbols) as a function of the stimulus onset asynchrony of paired stimuli in Experiment 2. The mean response probabilities for the S1-alone and S2-alone presentations are shown by the broken lines connecting the closed and open symbols, respectively. Error bars show \pm 1 SEM.

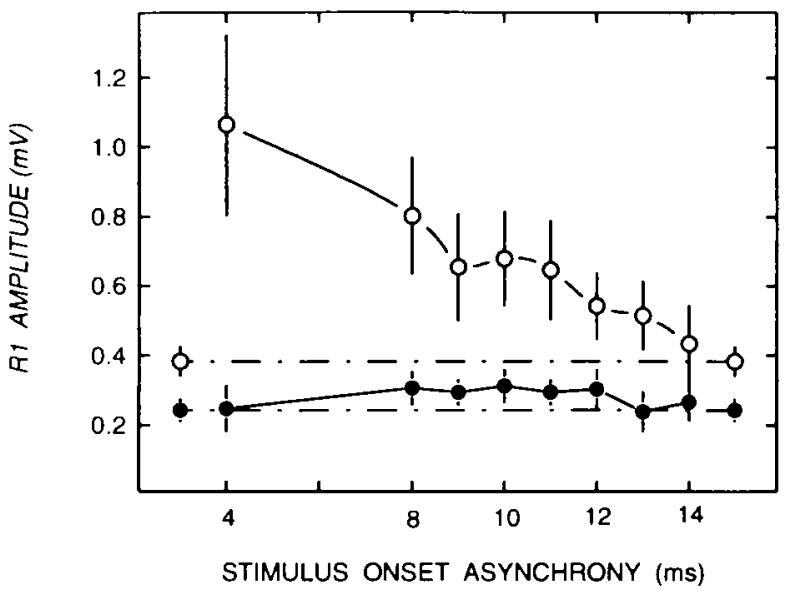

Figure 6. Mean response amplitude (in millivolts) of $\mathrm{R}_{1}$ (filled symbols) and $R 1_{2}$ (open symbols) as a function of the stimulus onset asynchrony of paired stimuli in Experiment 2. The mean response amplitudes for the S1-alone and S2-alone presentations are shown by the broken lines connecting the closed and open symbols, respectively. Error bars show \pm 1 SEM.

ing stimulus was greatest at the shortest SOA and decayed with SOA. The presence of reflex augmentation with S1 below threshold for reliable reflex elicitation implies that it is a consequence of processes initiated by the first stimulus and is not dependent on processes associated with emission of the first response. This implication was supported by the similarity of mean $R 1_{2}$ amplitude on trials on which an $\mathrm{R}_{1}$ was present $(M=.62 \mathrm{mV}, S E M=.04)$ and on trials on which $R 1_{1}$ was not present $(M=.53 \mathrm{mV}$, $S E M=.05$ ). The variable of $\mathrm{R} 1_{1}$ presence accounted for only $2 \%$ of the variance in $\mathrm{Rl}_{2}$ amplitude.

The first two experiments are alike in showing a systematic decay of augmentation with SOA. The absence of substantial decay of $R 1$ augmentation with an increase in SOA from 4 to $8 \mathrm{msec}$ in the report by Hammond and Dennon (1991) is attributable to 1 subject for whom large and constant responses were recorded at all SOAs; this appears to have been due to overamplification of the EMG with consequent clipping of response amplitude.

\section{EXPERIMENT 3}

Experiment 3 was designed to learn more about the dependence of reflex augmentation on the intensity of each member of the stimulus pair. The effects of three intensities of each member of a stimulus pair at 4- and 8-msec SOAs on the amplitude and latency of the R1 responses were examined.

\section{Method}

Subjects. Six volunteers were selected from the same population, with the same criteria as in Experiment 1;2 had served in both previous experiments, and 2 had served in one of the previous experiments. There were 4 males and 2 females, from 18 to 46 years old, with a median age of 20 years. 
Apparatus and Procedure. The procedures were similar to those in Experiment 1. Stimulation and recording procedures were the same as those in the previous experiments. The impedance between the recording electrodes ranged from 3 to $9 \mathrm{k} \Omega$ for all subjects. The thresholds were determined as in Experiment 1. The reliable elicitation threshold intensities for the 6 subjects ranged from 2.6 to $8.0 \mathrm{~mA}$, with a mean of $5.4 \mathrm{~mA}$.

Stimulus pairs were presented according to the factorial combination of three $\mathrm{S} 1$ intensities $(1.0,1.2$, and 1.4 times the $\mathrm{R} 1$ elicitation threshold), three $S 2$ intensities $(0.8,1.0$, and 1.2 times the R1 elicitation threshold), and two SOAs (4 and $8 \mathrm{msec}$ ). The session was divided into nine blocks, with each block devoted to a particular combination of S1 and S2 intensity; the successive blocks were separated by several minutes. Sixteen trials were run in each block: eight paired-stimulus trials at the specified combination of stimulus intensities at the two SOAs (4- and 8-msec) and eight singlestimulus trials, four at each of the two stimulus intensities used in the paired stimulus presentations. There were therefore a total of 144 trials given in each session, which lasted about $60 \mathrm{~min}$. The presentation order of the blocks within each session was random. The intertrial interval varied from 10 to $32 \mathrm{sec}$ with a mean of $16 \mathrm{sec}$.

Trials contaminated with EMG activity in the $10 \mathrm{msec}$ before the onset of the first stimulus were discarded; $1.0 \%$ of all trials were discarded in this way.

\section{Results and Discussion}

Two distinct R1 components were present on $75.4 \%$ of scoreable paired-stimulus trials on which R1 activity was detectable. The mean probability of elicitation of $R 1_{1}$ with paired stimuli is shown as a function of $\mathrm{S} 1$ intensity for each of the two SOAs in the top panel of Figure 7 and for $R 1_{2}$ as a function of $S 2$ intensity at each SOA in the bottom panel of the figure; the mean probability of elicitation of $R 1$ as a function of stimulus intensity with single-stimulus presentations is shown for comparison in both panels. The mean response probability with singlestimulus presentation increased linearly with stimulus intensity from $0.56(S E M=.07)$ at the lowest intensity to $0.99(S E M=.01)$ at the highest. The probability of elicitation of $R 1_{1}$ was affected by $S 1$ intensity (estimated $\omega^{2}=$ 0.22 ) but not by $S 2$ intensity (estimated $\omega^{2}=0.01$ ); conversely, the probability of elicitation of $R 1_{2}$ was affected by $S 2$ intensity (estimated $\omega^{2}=0.21$ ) but not by $S 1$ intensity (estimated $\omega^{2}=0.01$ ). The $\mathrm{R} 1_{1}$ means presented in Figure 7 are averaged over the $S 2$ intensities and the $R 1_{2}$ means are averaged over the $S 1$ intensities. These results confirm and extend the suggestion from the first two experiments that the two separate $\mathrm{R} 1$ responses are under independent control of the two successive stimuli.

The mean probability of eliciting $\mathrm{R} 1_{1}$ (top panel, Figure 7) with paired stimuli at both the 4- and the 8-msec SOA was similar to single-stimulus $\mathrm{R} 1$ probabilities. The appearance of the second stimulus did not affect the probability that the first elicited an R1. The probability of an $\mathrm{R} 1$ 's being elicited by the second stimulus (bottom panel) was, in contrast to that for $R 1_{1}$, affected by the presence of the first stimulus. The effect of the first stimulus is shown by the modest elevation of response probability with paired stimuli above the single-stimulus values. The elevation of response probability was generally greater

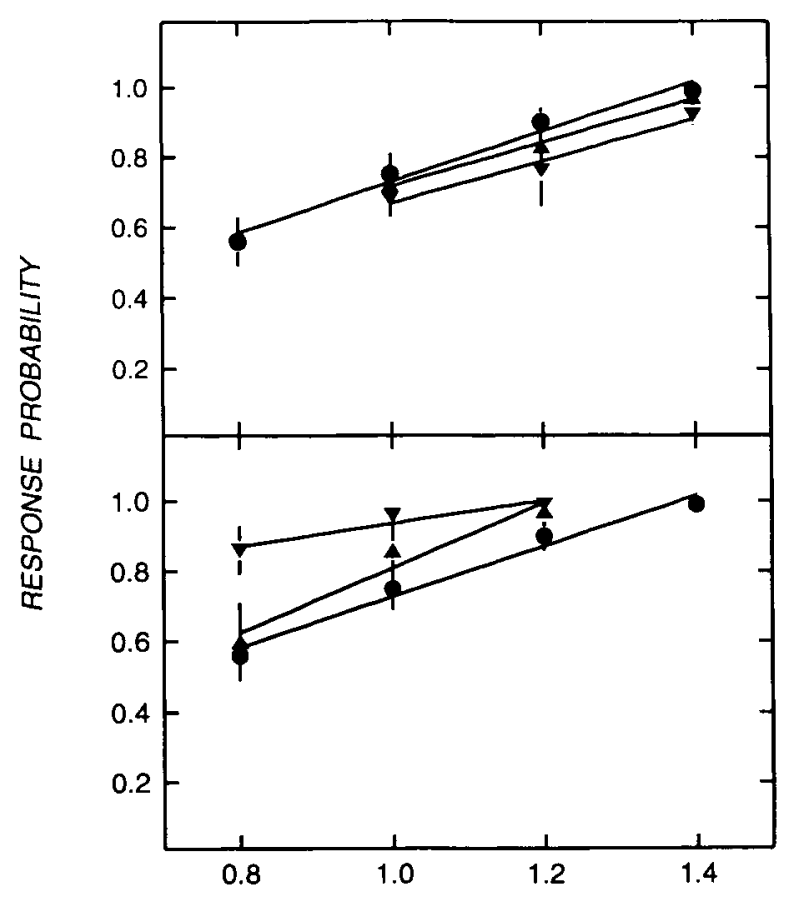

ELICITING STIMULUS INTENSITY (re threshold)

Figure 7. Mean response probability of $\mathrm{R1}_{1}$ (upper panel) and $\mathbf{R I}_{2}$ (lower panel) as a function of the eliciting stimulus intensity (S1 intensity for $R 1_{1}$ and $S 2$ intensity for $R 1_{2}$ ) with paired stimuli with 4-msec (inverted triangles) and 8-msec (upright triangles) stimulus onset asynchronies. The means for paired-stimulus presentation in the upper panel are averaged over $\mathbf{S 2}$ intensities; those in the lower panel are averaged over $S 1$ intensities. Mean single-stimulus response probabilities (filled circles) are repeated in each panel for comparison with the paired-stimulus probabilities. Best-fitting straight lines have been fitted to the data points.

at the 4- than at the 8-msec SOA, but the effect of SOA was not powerful, as is shown by the small proportion of variance that it accounted for (estimated $\omega^{2}=0.05$ ).

Mean R1 amplitudes from trials on which responses were recorded show the same effects as does response probability but more clearly. Mean amplitudes of the first and second responses as a function of the intensity of the first and second stimulus, respectively, are shown in the top and bottom panels of Figure 8, which also shows mean response amplitudes to single-stimulus presentations for comparison. The $R 1_{1}$ means are averaged over the $S 2$ intensities and the $R 1_{2}$ means are averaged over the $S 1$ intensities. Mean amplitude of $R 1_{1}$ with paired stimuli increased with S1 intensity at both SOAs (top panel, Figure 8). The mean response amplitudes were similar at both SOAs (4 and $8 \mathrm{msec}$ ) and did not vary much from single-stimulus R1 amplitudes. S1 intensity accounted for a substantial proportion of the variance (estimated $\omega^{2}=$ 0.19 ), but neither S2 intensity nor SOA was important (estimated $\omega^{2}=0.00$ and 0.01 , respectively).

In contrast, mean amplitudes of $R 1_{2}$ with paired stimuli were greater than single-stimulus amplitudes at each 


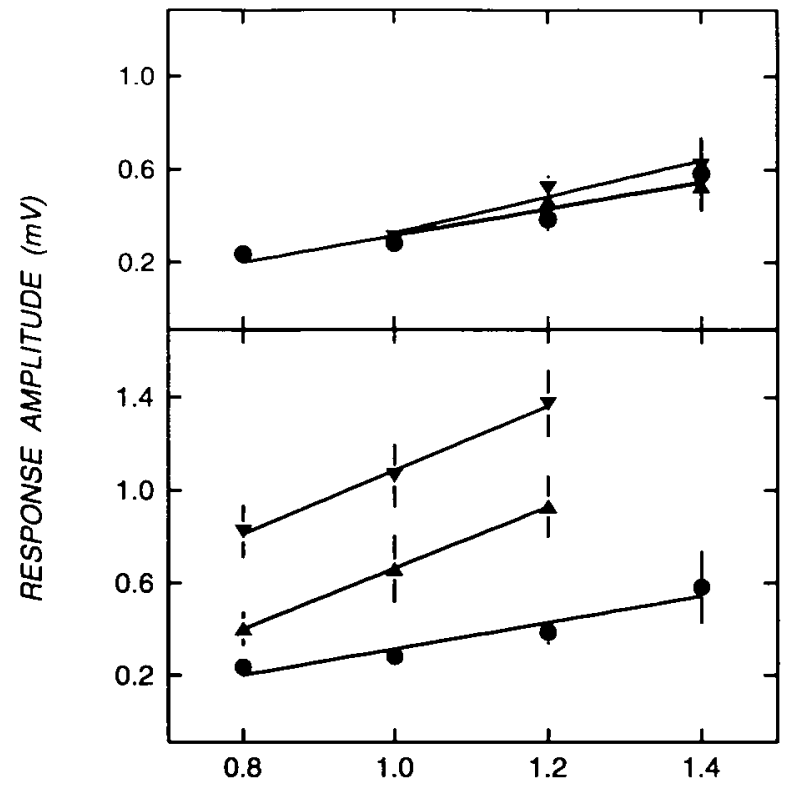

ELICITING STIMULUS INTENSITY (re threshold)

Figure 8. Mean response amplitude (in millivolts) of $\mathrm{R}_{1}$ (upper panel) and $\mathrm{RI}_{2}$ (lower panel) as a function of the eliciting stimulus intensity ( $S 1$ intensity for $R 1_{1}$ and $S 2$ intensity for $R 1_{2}$ ) with paired stimuli with 4-msec (inverted triangles) and 8-msec (upright triangles) stimulus onset asynchronies. The means for paired-stimulus presentation in the upper panel are averaged over S2 intensities; those in the lower panel are averaged over S1 intensities. Mean singlestimulus response amplitudes (filled circles) are repeated in each panel for comparison with the paired-stimulus amplitudes. Bestfitting straight lines have been fitted to the data points.

of the three $S 2$ intensities. This augmentation of response amplitude above single-stimulus levels with paired stimuli was greater at the 4-msec than at the 8-msec SOA. Furthermore, response augmentation increased with S2 intensity, shown by the steeper slopes of the response amplitude functions for paired than for single stimuli. These results can be accounted for by assuming that the first stimulus establishes potential augmentation that decays with time and is exploited to a greater extent by more intense second stimuli. For $\mathrm{R}_{2}, \mathrm{~S} 1$ intensity was not important (estimated $\omega^{2}=0.00$ ), but both $S 2$ intensity and SOA were (estimated $\omega^{2}=0.23$ and 0.29 , respectively). These results confirm that $R 1_{1}$ and $R 1_{2}$ are separate responses each under independent control of the two successive stimuli, and that with paired stimuli only the second response is augmented.

There were no important effects of the experimental conditions on response latency. The overall mean latencies were $11.5 \mathrm{msec}(S E M=.10)$ and $11.6 \mathrm{msec}$ $(S E M=.11)$ for the first and second responses, respectively.

\section{GENERAL DISCUSSION}

These experiments give a more detailed description of the effects of closely spaced percutaneous stimuli on the
$\mathrm{R} 1$ component of the blink reflex than was previously available. Two successive stimuli with SOAs as brief as 4 msec elicit successive R1s; the probability and amplitude of the response to the second stimulus are both increased by the first stimulus, but the response to the first stimulus is unaffected by the second stimulus. This result indicates that stimulation of the supraorbital branch of the trigeminal nerve, whether above or below threshold for R1 elicitation, produces a transient increase in the excitability of the $R 1$ pathway that decays progressively over about $12 \mathrm{msec}$. The increase in excitability was independent of stimulus intensity over the range of intensities used in Experiment 3. The transient R1 augmentation seen here corresponds to the first phase of the R1 recovery cycle with paired stimuli noted by Penders and Delwaide (1973). The brief duration of the augmentation produced by the leading $\mathrm{S} 1$ (about $12 \mathrm{msec}$ from the present data) means that it is unlikely to be a mechanism of the $R 1$ augmentation seen in other conditions, such as presentation of a preliminary stimulus with lead times of $50 \mathrm{msec}$ and longer (Sanes \& Ison, 1979) and with stimulus repetition with interstimulus intervals of 1,2 , and $5 \mathrm{sec}$ (Sanes \& Ison, 1983).

The augmentation of $\mathrm{R} 1$ seen with paired stimuli does not reflect the summation of stimulus energy. In these experiments, the probability and size of $R 1$ were determined by the characteristics of the eliciting stimulus and by the presence of a percutaneous stimulus just before the eliciting stimulus. In contrast to the conclusion of Hammond and Dennon (1991), it appears that the simple Rl pathway does not support temporal integration of stimulus energy. The restriction of augmentation to the response elicited by the second stimulus of a pair, together with its time course, makes it most readily understandable as the effect of a subliminal fringe of partially excited nerve cells created by the first stimulus (see, e.g., Henneman, 1974). The finding that the amount of augmentation increased with the intensity of the second stimulus is explicable as more of the subliminally excited cells are brought to discharge. This mechanism of the subliminal fringe has also been used to explain the augmentation of the acoustic startle reflex in rats seen when a nonreflexogenic stimulus precedes the reflex-eliciting stimulus by up to several milliseconds (Hammond \& Leitner, 1990).

In contrast to the $\mathbf{R} 1$ component, the later $\mathbf{R} 2$ does show temporal integration of successive stimuli in the sense that the R2 elicited by the first stimulus of a pair is augmented by a second stimulus that appears in the R2 latency before the emergence of the response (Plant \& Hammond, 1989). The difference in plasticity of the two components is presumably a function of the complexity of the neural pathway of each component; the more complex polysynaptic pathway of $\mathbf{R} 2$ supports temporal integration of stimulus energy, whereas the relatively simple pathway of R1 does not.

$R 1$ augmentation was evident both in an increased probability that the second stimulus of a pair elicited a response and in the size of the responses that were elicited by the second stimulus. It is not yet known whether this aug- 
mentation enhances the protective function of the blink reflex by facilitating lid closure. No concurrent measures of $R 1$ size and lid closure in conditions that augment $R 1$ have yet been reported. The propensity of $R 1$ to show augmentation in different conditions (see, e.g., Boelhouwer, Gregoric, van den Bosch, Schomaker, \& Brunia, 1982; Ison et al. 1990; Sanes \& Ison, 1983) and the observations that $\mathrm{R} 1$ can initiate lid closure (Snow \& Frith, 1989; Sunohara, Tomi, Satoyoshi, \& Tachibana, 1985) suggest that $\mathrm{R} 1$ augmentation may be behaviorally important.

\section{REFERENCES}

Boelhouwer, A. J. W., Gregoric, M., van den Bosch, W. E. J., SChomakeR, L. R. B., \& BRUNia, C. H. M. (1982). Habituation of the blink reflex: The effect of stimulus frequency and the state of arousal. Physiological Psychology, 10, 325-330.

Evinger, C., Manning, K. A. (1988). A model system for motor learning: adaptive gain control of the blink reflex. Experimental Brain Research, 70, 527-538.

Hackley, S. A., \& Graham, F. K. (1984). Early selective attention effects on cutaneous and acoustic blink reflexes. Physiological Psychology, 11, 235-242.

Hammond, G. R., \& DENNon, S. (1991). Temporal integration shown in the early and late components of the human blink reflex. Psychobiology, 19, 180-186.

Hammond, G. R., \& Leitner, D. S. (1990). Augmentation of the rat's acoustic startle reflex by nonreflexogenic stimuli. Behavioral Neuroscience, 104, 841-848.

Henneman, E. (1974). Spinal reflexes and the control of movement. In V. B. Mountcastle (Ed.), Medical physiology (13th ed, Vol. 1, pp. 651-667). St. Louis: Mosby.

Ison, J. R., Sanes, J. N., Foss, J. A., \& Pinckney, L. A. (1990). Facilitation and inhibition of the human startle blink reflexes by stimulus anticipation. Behavioral Neuroscience, 104, 418-429.

Penders, C. A., \& Delwaide, P. J. (1973). Physiologic approach to the human blink reflex. In J. E. Desmedt (Ed.), New developments in electromyography and clinical neurophysiology: Vol 3. Human reflexes, pathophysiology of motor systems, methodology of human reflexes (pp. 649-657). Basel: Karger.

Plant, Y., \& Hammond, G. R. (1989). Temporal integration of acoustic and cutaneous stimuli shown in the blink reflex. Perception \& Psychophysics, 45, 258-264.

SANES, J. N., \& Ison, J. R. (1979). Conditioning auditory stimuli and the cutaneous eyeblink reflex in humans: Differential effects according to oligosynaptic or polysynaptic central pathways. Electroencephalography \& Clinical Neurophysiology, 47, 546-555.

SANES, J. N., IsON, J. R. (1983). Habituation and sensitization of components of the human eyeblink reflex. Behavioral Neuroscience, 97, 833-836.
SNOw, B. J., \& FRITH, R. W. (1989). The relationship of eyelid movement to the blink reflex. Journal of the Neurological Sciences, 91 , 179-189.

Sunohara, N., Tomi, H., Satoyoshi, E., Tachibana, S. (1985). Glabella tap sign: Is it due to a lack of R2-habituation? Journal of the Neurological Sciences, 70, 257-267.

VRana, S. R., SPEnce, E. L., LANG, P. J. (1988). The startle probe response: A new measure of emotion? Journal of Abnormal Psychology, 97, 487-491.

\section{NOTE}

1. Two difficulties were encountered scoring reflex amplitude on trials on which both $R 1_{1}$ and $R 1_{2}$ occurred. First, $R 1_{1}$ merged into $R 1_{2}$ at the short SOAs. On these trials, the first peak of $R l_{1}$ was unaffected and easily scored, but with a 4-msec SOA, the second peak had to be estimated by visual extrapolation of the early part of the $R 1_{1}$ waveform. Similarly, the second peak of $R 1_{2}$ was unaffected, but the first peak had to be estimated by extrapolation. Trials without overlap of $R l_{1}$ and $R 1_{2}$ showed that for individual subjects the latency of each $R 1$ was stable and that there was little variation in their duration, ranging typically from 7 to $10 \mathrm{msec}$. These observations guided the extrapolations by defining narrow zones in which the extrapolations of onsets and peaks were made. The slight overlap of $R 1_{1}$ and $R 1_{2}$ at the 8 -msec and longer SOAs did not affect scoring because the largest positive and negative deflections of $R l_{1}$ (from which its peak-to-peak amplitude was scored) were complete by the onset of $R 1_{2}$. The stability of the scored $R 1_{1}$ peak amplitude at the different SOAs (see Figure 4) does not suggest a systematic scoring bias at the short SOAs. Second, $R 1_{1}$ was partly obscured by the artifact created by $S 2$ in the EMG recordings at the long SOAs. This made small responses difficult to detect and was probably responsible for the apparent suppression of $R 1_{1}$ probability at the long SOAs seen in Figure 3. The positive- and negative-going peaks of the larger $R 1_{1}$ responses were extrapolated from the signal that was free from stimulus artifact (the duration of the stimulus artifact was always less than $2 \mathrm{msec}$ ); the slower rate of change of the $R 1_{1}$ waveform made it easy to distinguish from the rapid rate of change of the stimulus artifact. The difficulties faced in scoring the latency of each component were resolved in a similar way. At the short SOAs, the onset of $R 1_{2}$ was extrapolated from the later part of the waveform; the extrapolation was straightforward, because at these SOAs $R 1_{2}$ was usually larger than $R 1_{1}$ and the rate of change of the EMG trace was correspondingly greater. At the long SOAs, the onset of $R 1_{1}$ was extrapolated when it was obscured by the stimulus artifact of $\mathrm{S} 2$; the short duration and rapid rate of change of the stimulus artifact made it readily distinguishable from the $\mathrm{R} 1_{1}$ waveform.

(Manuscript received December 4, 1991; revision accepted for publication October 12, 1992.) 